\title{
EL CONCEPTO DE CICLO HIDRO-SOCIAL APLICADO A LOS CONFLICTOS POR EL ACCESO AL AGUA. EL CASO DE LA DISPUTA POR EL RÍO ATUEL ENTRE LAS PROVINCIAS DE LA PAMPA Y MENDOZA, ARGENTINA
}

\author{
María Laura Langhoff ${ }^{\prime}$, Alejandra Geraldi ${ }^{2}$ \& Patricia Rosell ${ }^{3}$ \\ Universidad Nacional del Sur (UNS)
}

\section{RESUMEN}

El presente artículo plantea un abordaje del concepto de ciclo hidro-social desde la perspectiva de la ecología política del agua. Este análisis teórico facilita el estudio del conflicto por el río Atuel, entre las provincias de La Pampa y Mendoza, Argentina, para explicar a través de una matriz los elementos que interactúan en el ciclo hidro-social y determinar las consecuencias que estos generan.

El objetivo es analizar el ciclo hidro-social y su aplicación al problema para un sector del oeste pampeano cuyo eje organizador es el río Atuel. Así mismo, estudiar los conflictos interprovinciales que se generan y los aspectos que componen dicho ciclo.

El tratamiento del problema y su profundización es abordado a través de la lectura de trabajos de especialistas en el área y de las fuentes hasta ahora analizadas tales como: mensuras catastrales, testimonios de pobladores y documentación oficial.

Palabras claves: ciclo hidro-social; ecología política; agua; conflicto; oeste pampeano.

\section{THE CONCEPT OF HYDROSOCIAL CYCLE APPLIED TO THE CONFLICTS CAUSED BY ACCESS TO WATER. THE CASE OF THE DISPUTE BETWEEN THE ARGENTINE PROVINCES OF LA PAMPA AND MENDOZA OVER ATUEL RIVER}

\section{ABSTRACT}

This article offers an approach to the concept of hydro-social cycle from the perspective of the political ecology of water. This theoretical analysis facilitates the study of the conflict between the Argentine provinces of La Pampa and Mendoza over Atuel River using a matrix to explain the elements that interact in the hydro-social cycle and determine the consequences that they generate.

The goal is to analyse the hydro-social cycle and its application to the problem in an area located in the west of the province of La Pampa, whose organizing axis is Atuel River. Likewise, it aims at studying interprovincial conflicts which are generated and the aspects which make up the above mentioned cycle.

The problem is dealt with and analysed in depth through the reading of the work of specialists in the area and the sources which have been analysed up to now such as: cadastral measures, testimonies of inhabitants and official documents.

Key words: hydrosocial cycle; political ecology; water; conflict; Pampean west.

\footnotetext{
1 Departamento de Geografía y Turismo, Universidad Nacional del Sur. E-mail: laura.langhoff@outlook.com.

Departamento de Geografía y Turismo, Universidad Nacional del Sur; CONICET. E-mail: ageraldi@criba.edu.ar.

33 Departamento de Geografía y Turismo, Universidad Nacional del Sur. E-mail: prosell@uns.edu.ar.
} 


\section{INTRODUCCIÓN}

Desde fines del siglo pasado la disponibilidad de agua dulce se ha visto alterada por la variabilidad en los ciclos climáticos y, a su vez, por el uso intensivo del recurso con fines diversos, como consecuencia se produjo un agravamiento de los conflictos para acceder a ella.

Según World Resources Institute para el año 2040 los países con stress hídrico a nivel mundial serán aquellos localizados en la zona ecuatorial del planeta y en sectores con escasas precipitaciones, como: Bahréin, Kuwait, Palestina, Qatar, San Marino, Singapur, Emiratos Árabes Unidos, Israel, Arabia Saudita, Omán y Líbano ${ }^{4}$ (LUO, YOUNG y REIG, 2015). En este esquema a futuro, la zona del Mediterráneo, que además recibe la presión del turismo masivo, también se verá afectada, como el caso de la costa española, donde ya se han dado algunos avances en cuanto a la gestión del agua (MOROTE SEGUIDO, 2016; MOROTE, SAURÍ y HERNANDEZ, 2016; OLCINA CANTOS y VERA REBOLLO, 2016a y 2016b). En el caso de Latinoamérica y en Argentina, la alarma por el uso desmedido de agua dulce se relaciona con la sobreexplotación del recurso para satisfacer las demandas del modelo neo-extractivista ${ }^{5}$, impulsado desde fines de los años ochenta en el marco de la instrumentación de políticas neoliberales y aún vigente por medio de actividades como la megaminería y los monocultivos industriales (PERALES MIRANDA, 2010; YACOUB, DUARTE y BOELENS, 2015; IVARS, 2015).

En este contexto se generan conflictos, donde adquieren importancia diversos sectores de la sociedad, para MERLINSKY (2013) son en cierto modo una herramienta que permite la "discusión sobre estados de mundos posibles" entre distintos sujetos que suman, además, demandas y respuestas tentativas. En relación al agua los conflictos pueden ser diferenciables. Por un lado, los que se producen en medios urbanos donde el abastecimiento de agua para toda la población se altera por distintos intereses y factores. Y por otro, aquellos que afectan las economías de los espacios rurales derivados de la alteración del curso de los ríos, contaminación de las aguas, amenazas de inundaciones entre tantos.

En estos conflictos hay dos posiciones con intereses opuestos de parte de los actores involucrados: por un lado, quienes necesitan y reclaman el agua para vivir ${ }^{6} \mathrm{y}$, por otro lado, quienes aprecian o consideran el agua como un bien de valor económico para generar riqueza desde un posicionamiento netamente capitalista y productivista. Del mismo modo, los espacios rurales entran en conflicto por el agua ante la construcción de canales clandestinos fuera de toda gestión y control y potencian el conflicto en sí mismo.

Estos conflictos inducen a nuevas perspectivas de análisis, entre ellos, los estudios en el campo de la ecología política, los cuales han abierto una línea de investigación denominada ecología política del agua, que se caracteriza por su interdisciplinariedad, siendo los referentes SWYNGEDOUW (2009, 2013, 2015), LINTON (2010), BOELENS (2015, 2016), y algunos trabajos como los de ILLICH (1974). El eje de este campo, se articula en las relaciones de poder que establecen los actores diversos en el proceso de apropiación de este recurso o bien común.

El aporte de SWYNGEDOUW (2009) se basa en utilizar el concepto de geometrías de poder en referencia a las estrategias que se ponen en juego en el proceso de distribución del agua: "The political-ecological examination of the hydro-social process reveals the inherently conflict-ridden nature off the process of socio-environmental change and teases out the

\footnotetext{
${ }^{4}$ Surgen algunos países que han logrado un crecimiento rápido en las últimas décadas, vinculados a la explotación petrolera y a la expansión financiera. Un ejemplo son los E.A.U. donde además ha aumentado la población y las actividades ligadas a turismo y servicios financieros, lo que ha elevado las demandas de agua potable y para riego con el fin de producir de alimentos y el mantenimiento de los espacios verdes urbanos. Para lograr este abastecimiento se han construido unas 100 plantas desalinizadoras y se planean nuevas técnicas para obtener agua en un medio signado por la aridez. No obstante, el funcionamiento de estas plantas está generando cuestionamientos dado que funcionan con carbón que es un alto contaminante ambiental.

${ }^{5}$ Continuación del modelo extractivista colonial iniciado en 1492.

${ }^{6}$ Estos pueden realizar su reclamo generalmente desde una lógica inserta en el lugar, considerando a éste desde su significancia antropológica y social (ESCOBAR, 2010).
} 
inevitable conflicts (...) It is these power geometries and the social actors carrying them that ultimately decide who will have Access to or control over, and who will be excluded from Access to or control over, resources or other components of the environment" (SWYNGEDOUW, 2009, 57).

El autor relaciona la circulación del agua en medios urbanos y los flujos de poder asociados, aspectos que resultan claves para entender al ciclo del agua como la conjunción de eventos donde intervienen elementos sociales y propios de la naturaleza como la variabilidad climática. Con esta base se debe contemplar que el ser humano está actuando constantemente sobre el medio natural $\mathrm{y}$, puntualmente, en cuanto al acceso al agua a través de la aplicación de una determinada tecnología. De este modo, se usan distintas técnicas para el aprovechamiento tanto de las aguas disponibles a nivel superficial como subterráneo (las napas y acuíferos), por medio de la construcción de diques, presas, canales, produciendo inundaciones, desviando cursos de agua o disecando lagunas y pantanos.

En su conceptualización del ciclo hidro-social, SWYNGEDOUW (2009) plantea, además, que éste puede ser entendido como el proyecto por medio del cual se configura una organización socio-ambiental en torno al agua con el objetivo (hoy en día desde la ecología política) de que se logre un acceso más democrático al recurso. Históricamente se pueden identificar múltiples ciclos hidro-sociales de acuerdo al lugar (región, país, continente), los cuales han respondido a diversos intereses y tecnologías. En su desarrollo es posible estudiar los juegos de poder y tensiones que se han ido gestando y manifestando. Por otro lado, el autor afirma lo siguiente "Landless, peasants, smallholders and large landowners alike, workers and capitalist, political affiliations and visions of what is right or wrong, a wide range of institutional constellations, geopolitical tensions and alliances, the heterogeneous physical and ecological acting of water and all sorts of other non humans things and organisms, and much more, coalesce in the production of hydroscape, the hydro-social assemblage that capture, transforms, metabolizes, moves and distributes to quench the thirst of cities, to fertilize the fields and to permit all manner of other production processes to unfold" (SWYNGEDOUW, $2015,24)$. En base a estos aportes, el ciclo hidro-social es un concepto instrumental que permite abordar el problema del acceso al agua y su distribución, desde una perspectiva que contempla la complejidad y cuestiona conceptos e ideas dados y utilizados como términos universales. Tal como sucede con el caso de "agua" y "naturaleza" tal cual lo plantea SWYNGEDOUW (2011), cuando afirma que "la naturaleza no existe".

En relación con las tensiones y conflicto, LARSIMONT (2014) agrega al análisis del ciclo hidro-social las estrategias por medio de las cuales el agua es manipulada, no solo por medio de infraestructura hídrica, sino por la elaboración de marcos legales, la influencia de las instituciones y las prácticas culturales "la circulación del agua pone en evidencia procesos políticos, económicos, sociales y ecológicos a diferentes escalas" (LARSIMONT, 2014, 4).

De acuerdo a las prácticas productivas que se realicen y el grado de las intervenciones humanas en la circulación del agua a través del tiempo, se definirán los ciclos hidro-sociales. En algunos casos serán armoniosos y en otros se producirán desequilibrios que afectarán al medio ambiente y a la sociedad involucrada.

Últimamente los niveles de contaminación han llegado a la atmósfera afectando al agua en estado gaseoso e incluso alterando el clima en lo que se conoce como "cambio climático", producto, también, de otros procesos de degradación ambiental, provocando inundaciones y sequías en lugares donde antes no se presentaban estas problemáticas.

Retomando el concepto de la ecología política, su conocimiento enriquece el camino hacia la justicia del agua y los desequilibrios ligados a su acceso en el mundo, específicamente en América Latina. El por qué, es evidente debido a que se logran identificar los actores

\footnotetext{
${ }^{7}$ El estudio "Cambio climático y diversidad biológica" realizado por Naciones Unidas y PNUMA en el año 2007, expone dentro de los efectos que deberán esperarse a futuro una disminución en las precipitaciones en el centro de Asia, la zona del Mediterráneo, África, partes de Australia y Nueva Zelanda; desaparición de hielo marino por aumento de temperaturas, entre otros efectos que ya se están iniciando. El proceso ya es irreversible, si de modo hipotético se detuvieran en este momento las emisiones, los efectos ya están en marcha.
} 
involucrados, las tensiones e intereses puestos en juego y los discursos y representaciones que se esgrimen en el proceso de apropiación y control del recurso. Al respecto, BOELENS (2015) plantea la importancia de considerar la utilización racional del agua por parte de los campesinos en las zonas andinas, cimentada en las tradiciones transmitidas en algunos casos desde épocas pre-hispánicas. Estas prácticas se contraponen a las intervenciones y sugerencias de "expertos" cuyos conocimientos y experiencia está sustentada en otra lógica de carácter productivista: "Throughout Latin America, growing demand and declining availability bring about escalating water conflicts. Inequality is very deep indeed. From Argentina to Mexico, water is reduced to an economic resource allocated to the 'most profitable users and uses' in the win-or-losemarket. Water dispossession is now tan ever" (BOELENS, 2015, 9).

YACOUB et al., (2015) incorpora el concepto de "territorio hidro-social" el cual busca definir los espacios que involucra el modelo económico extractivista hegemónico en el continente, en los que se sobreexplotan las disponibilidades de agua, como son la megaminería, los monocultivos, la expansión de cultivos sobre tierras áridas con riego intensivo y el fracking, entre otras. Un territorio hidro-social debe ser entendido como el espacio revalorizado por la presencia de agua que resulta esencial para las actividades productivas, pero que también tiene un papel importante a nivel cultural. El agua pasa a ser un lazo de relación con el territorio y, en torno a su existencia, se articulan las demás actividades y representaciones. Esta conceptualización se completa con el aporte de BOELENS, et al., (2016) quienes en un reciente trabajo lo definen como: "Territories although often considered natural, are actively constructed and historically produced through the interfaces amongst society, technology and nature. They are the outcomes of interactions in which the contents, presumed boundaries and connections between nature and society are produced by human imagination, social practices and related knowledge systems. This is clearly manifested in how river basin management, water flows, water flows use systems and hydrological cycles are mediated by governance structures and human interventions that entwine the biophysical, the technological, the social and the political" (BOELENS, et al., 2016, 2).

Estos conceptos presentados permiten guiar el estudio de la problemática del acceso al agua en el oeste pampeano y establecer los aspectos de análisis adecuados. Estos son: los actores, los imaginarios entorno al agua, las tensiones, el conflicto y el territorio hidro-social específico. Estos estructuran el proceso de tensión y conflictividad a lo largo del siglo XX e inicios del XXI, período en que se va conformando un nuevo ciclo hidro-social enmarcado en un modelo económico de producción capitalista orientado a la exportación. En base a lo expuesto el objetivo del presente trabajo es analizar el ciclo hidro-social y su aplicación al problema para un sector del oeste pampeano de Argentina. Así mismo, estudiar los conflictos interprovinciales que se generan y los aspectos que componen dicho ciclo. Se pretende contribuir al aporte de una perspectiva en este conflicto, que permita, a futuro, delinear una alternativa de solución que pueda además ser útiles en conflictos similares.

El trabajo hace especial hincapié en los usos actuales del agua tanto en Mendoza como La Pampa dentro del territorio hidro-social. Así, es posible esquematizar el ciclo hidro-social en un contexto de escasez y conflicto. Las propuestas aportan a una comprensión más abarcativa del problema para contribuir a una posible solución.

\section{METODOLOGÍA}

La metodología utilizada se basa en la triangulación de fuentes tanto cuantitativas (estadísticas) con fuentes cualitativas (reclamos, cartas, cartografía, informes y notas periodísticas). Esta metodología consiste en la combinación de teorías, fuentes de datos, métodos de investigación, en el estudio de un fenómeno singular. La lectura crítica y contraste de los estudios realizados sobre la población y ocupación del sector de estudio, aportan los datos sobre los períodos de asentamiento de población y aquellos en los que comienza a retraerse. Este es un análisis que comprende la escala provincial que a su vez, se contrasta con las fuentes y bibliografía que abordan el área a nivel interprovincial. Las ventajas que presenta esta metodología es que cuando dos estrategias arrojan resultados similares, se corrobora el fenómeno estudiado; pero cuando los resultados no lo son, se presenta la oportunidad para 
elaborar una nueva perspectiva, es posible así, señalar la complejidad del objeto de estudio y brindar la oportunidad de que se realicen nuevos planteamientos. Como resultado de esta triangulación, se definió el ciclo hidro-social y se obtuvieron los aspectos que permiten analizarlo en este caso particular.

\section{CARACTERIZACIÓN DEL ÁREA DE ESTUDIO}

El río Atuel es un río interprovincial, dado que se forma en la provincia de Mendoza y discurre hasta la vecina provincia de La Pampa (Figura 1). Conforma la cuenca Desaguadero, una de las más extensas del país, ésta se forma en la provincia de La Rioja y corre en dirección sur con una extensión de unos $1000 \mathrm{~km}$. En su trayecto recibe el aporte de siete ríos principales alimentados por los deshielos de la cordillera de los Andes: Bermejo, Jachal, San Juan, Mendoza, Tunuyan, Diamante y Atuel. Los cuatro primeros ríos alcanzan su nivel de base en las lagunas de Guanacache, en tanto los otros tres lo hacen en el río Desaguadero-SaladoChadileuvú que, a su vez, lo alcanzan en las lagunas pampeanas de La Dulce, La Amarga y Urre Lauquen. La característica de esta cuenca es que es endorreica, es decir, no tiene salida al mar, solo en ocasiones de excepcionales aportes de agua, el complejo de lagunas pampeano ha desbordado aportando aguas al río Curaco, para de este modo desaguar en el río Colorado y de allí al océano Atlántico.

FIGURA 1

Localización geográfica del área de estudio en el oeste pampeano, ríos Atuel y Salado

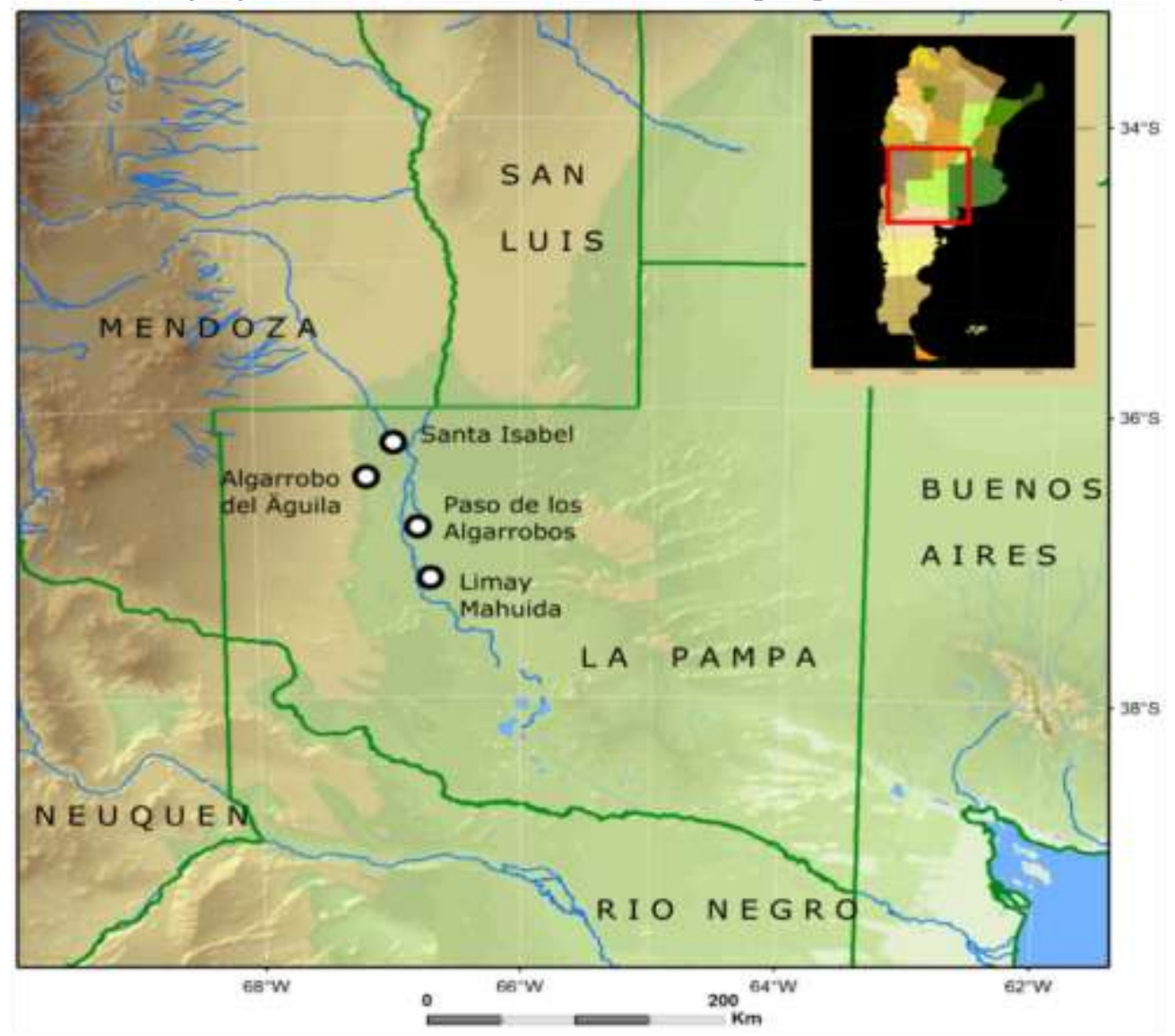

Fuente: Elaboración propia.

La existencia de este curso de agua favoreció históricamente al oeste pampeano al permitir el desarrollo de vegetación en un medio semiárido. Estudios arqueológicos realizados a mediados del siglo pasado, revelaron sitios con ocupación humana en tiempos históricos. Uno de ellos es 
el yacimiento arqueológico Vallejo, localizado a tres kilómetros de Santa Isabel, allí en un área de medanales se encontraron a inicios de los años sesenta, restos de huesos de animales autóctonos que sirvieron como alimento a la población indígena; instrumental lítico; trozos de cerámica y algunos elementos de época hispano-criolla (hierro, latón y vidrio). Debido a que en esa época los trabajos arqueológicos se estaban iniciando en La Pampa, no se pudo fechar fehacientemente ni establecer comparaciones con otros sitios, no obstante, el arqueólogo que llevó adelante los estudios creía que se correspondían aproximadamente, con el siglo XVI. En parte se basó en la existencia de material hispano entre los restos rescatados. De todos modos, este yacimiento es una evidencia de la presencia humana en esta zona.

Cuando el hombre vivió en el sitio Vallejo el paisaje era desde el punto de vista geomorfológico, semejante en los aspectos fundamentales al actual, solo que el río corría con regularidad y los bañados estaban activos (AUSTRAL, 1971).

En las décadas de los setenta y ochenta con la construcción de la represa de Casa de Piedra sobre el río Colorado, se realizaron estudios y rescates arqueológicos en una amplia zona del suroeste pampeano. Estos estuvieron bajo la dirección de Carlos Gradin. Se trabajaron en tres unidades: sobre el río Curaco donde se hallaron 29 sitios y en la cuenca inferior del Chadileuvú donde se hallaron 12 sitios arqueológicos, por último Lihue Calel con 35 sitios dentro del área protegida (BERÓN, 2008). Algunos de los mismos fueron lugares ocupados recurrentemente y donde los grupos humanos vivieron gran parte del año. Los fechados de ocupación indican unos 9000 años de antiguiedad en el sitio Casa de Piedra 1, en tanto en la zona semidesértica surcada por el río Curaco evidencia presencia humana en un rango que va desde 5000 a 500 años antes del presente (sitio Tapera Moreira). En esta zona se encontraron enterratorios (sitios 3 y La Lomita) con antigüedades entre 3000 y 2500 años antes del presente. Los trabajos han permitido establecer que las condiciones semidesérticas muy semejantes a las actuales se definieron en el período del Holoceno tardío (BERÓN, 2008), con sequías periódicas. En este momento se iniciaría la ocupación del espacio interior atravesado por los ríos Chadileuvú y Curaco. Ello permite comprender la importancia de estos ríos, la circulación del agua que permitió la vida y el asentamiento de diversos grupos humanos desde tiempos prehispánicos hasta el periodo colonial. En suma este no fue un espacio deshabitado, el ser humano interaccionó con el medio ambiente. Por otra parte, los trabajos arqueológicos han sido variados y permitieron la reconstrucción del pasado de gran parte del territorio pampeano, al respecto PODUJE (2004) presenta un acercamiento a los distintos proyectos arqueológicos y de preservación del patrimonio en la provincia.

Cabe precisar que esta región ha estado expuesta a alternancias de períodos secos y húmedos al menos desde el Holoceno hasta el presente, coincidiendo estos cambios con la presencia humana en la región, al menos desde hace 10.000 años. En el tramo final de dicha cuenca y durante los ciclos húmedos, ya en la provincia de La Pampa, el río Atuel se une al Salado formando un delta debido a las características del terreno más llano ${ }^{8}$. Aquí ingresa a través de cinco brazos principales, cuando tres de estos brazos se reactivan el río ingresa en dirección oeste-este: Atuel (que se une con el Chadileuvú), Butalo, Arroyo de la Barda y los más pequeños llamados de la Tinajera y de los Ingenieros (Figura 1). Alrededor de los $35^{\circ} 30^{\prime}$ de latitud Sur existen evidencias de una extensa área de bañados de $220 \mathrm{~km}$. de extensión por 60 $\mathrm{km}$. de ancho, conocidos como "los Bañados del Atuel". Estos se caracterizaban por la presencia de islotes, cauces, canales y brazos secundarios, lagunas y humedales (CAZENAVE, 1995) que permitieron el desarrollo de fauna y flora, sobre todo pasturas, variadas en un área semidesértica. Esta zona de bañados desapareció como consecuencia del accionar humano sobre el curso superior del río Atuel (CAZENAVE, 1995), perjudicando a los pobladores pampeanos que aprovechaban este oasis natural para la crianza de ovinos y caprinos.

Actualmente esta región se localiza dentro de lo que se conoce como "diagonal árida Sudamericana" o "diagonal arreica". Esta se caracteriza por ser una extensa franja de escasas

\footnotetext{
${ }^{8}$ La unión de estos ríos formaba el Chadileuvú y Curaco, alimentando las lagunas La Amarga y La Dulce en cercanías del Parque Nacional Lihue Calel. Finalmente sus aguas se unían a las del río Colorado que desagua al Atlántico. Debido al corte del Atuel y la merma de los caudales del Salado, las aguas de estos ríos que regaban el centro oeste provincial semiárido, corren eventualmente.
} 
lluvias que discurre desde la zona de Perú hasta las costas patagónicas. En su extensión se desarrollan una serie de espacios áridos. Factores como la divergencia anticiclónica en las costas de Perú y norte de Chile, junto con la cordillera andina en el noroeste argentino, dificultan la circulación de masas de aire húmedas. Ya sobre la zona patagónica sucede algo similar con el cordón montañoso ubicado al oeste que impide el ingreso de la humedad. Este cordón actúa como una barrera por lo que los vientos ingresan como vientos secos. BRUNIARD (1982) afirma que a esta franja se la puede considerar tanto como una "unidad fisiográfica, es decir, como un espacio con caracteres propios derivados de su común aridez; o bien, dejando de lado su condición areal, puede ser tratada como un límite, sea lineal o como una franja más o menos angosta, pero con significación limitante más que espacial” (BRUNIARD, 1982, 5).

\section{RESULTADOS Y DISCUSIÓN}

\subsection{OFERTA Y DEMANDA HÍDRICA EN EL OESTE PAMPEANO}

La población del oeste pampeano en su mayoría rural está compuesta por criollos (término que surgió en la época colonial para nombrar a las personas nacidas en América que descendían exclusivamente de padres españoles o de origen español) y descendientes de pueblos originarios (denominación actual de los descendientes de los nativos antes de la llegada de los europeos, en este caso es el pueblo Ranquel). Las extensiones de las explotaciones rondan entre las 1000 ha. y 5000 ha. En muchos casos se han desempeñado como cuidadores de los campos por generaciones, en otros han adquirido permisos precarios (que no aseguran la propiedad de la tierra) o son arrendatarios. La tenencia de la tierra es precaria, este es una característica que se repite en muchos lugares del oeste.

Las situaciones en que viven muchas de estas familias son muy particulares, su espacio de vida en el campo gira en torno al "puesto", este es el espacio que nuclea la actividad doméstica y de cuidado de los animales (MEDUS y PODUJE, 1997). Los puesteros pueden ser caracterizados como campesinos de tierras marginales pampeanas, se dedican a la crianza de caprinos y, en menor medida, vacunos. Una parte de lo que producen lo comercializan a muy bajo costo, lo que no les permite elevar sus condiciones sociales.

El Estado provincial implementó programas en algunos casos en coordinación con otras instituciones como el INTA (Instituto Nacional de Tecnología Agropecuaria) sin embargo, no han dado resultados esperados. Uno de los motivos es que han sido, en cierta forma, impuestos sin que mediara previamente un dialogo profundo con los productores destinatarios y se atendiera sus intereses concretos. Algunos de estos programas son: Plan Ganadero del Oeste (1974) con el Consejo Federal de Inversiones; Proyecto de Desarrollo Integrado para Pequeños Productores del Extremo Oeste de La Pampa (1986) y Plan de Desarrollo del Oeste Pampeano (2000). En el último caso se orientó a la producción cárnica caprina con la apertura de un frigorífico cuyo resultado no fue positivo por los bajos precios adquiridos en las ventas.

En cuanto a las localidades y parajes, estos cumplen una importante función social dado que allí se localizan las escuelas y albergues (escuelas hogares), las salas de atención médica primaria y las comisiones de fomento que cumplen un rol importante en relación a la producción (tramites de guías, etc.). Esta región no cuenta con emprendimientos industriales o que impliquen una alta demanda de tecnología, por lo tanto el grado de innovación es bajo.

En la Tabla 1 es posible analizar cómo ha sido la evolución de la población urbana y rural, registrada en los censos tomando la mitad del siglo XX hasta el año 2001. 
TABLA 1

Tabla de evolución de la población en los departamentos afectados al estudio

\begin{tabular}{|l|r|r|r|r|r|r|r|r|r|}
\cline { 2 - 9 } \multicolumn{1}{c|}{} & \multicolumn{7}{c|}{ Año } \\
\hline Departamento & 1920 & 1935 & 1942 & 1947 & 1964 & 1970 & 1980 & 1991 & 2001 \\
\hline Chalileo & 1750 & 1888 & 2102 & 2420 & 2048 & & 1729 & 2097 & 2493 \\
\hline Chical Co & 769 & 1198 & 1037 & 1189 & 1211 & 916 & 1199 & 1191 & 1595 \\
\hline
\end{tabular}

Fuente: Elaboración propia en base a datos censales extraídos de DIFRIERI (1981) y a la proyección realizada por la Dirección General de Estadísticas y Censos (2008) ${ }^{9}$.

En relación a la problemática planteada, se suma el problema del agua en cuanto a demanda para el consumo. La oferta hídrica superficial en la zona de estudio se debe a la escorrentía temporaria del Arroyo de la Barda y Arroyo Butalo, dos de los brazos del Atuel, superficie conflictiva dada los escases del recurso. En tanto la disponibilidad de aguas subterráneas también cuenta con dificultades dado que las napas ${ }^{10}$ se encuentran a gran profundidad. En el departamento Chical Co está en explotación un pozo cuyo caudal es de 2,5 m cúbicos por hora $^{11}$. Como se puede apreciar las disponibilidades de agua dulce son limitadas. El reclamo por el acceso al agua se agrava con los períodos de sequía, momento en que el gobierno suele aprovisionar por medio de camiones cisternas a las poblaciones.

Sin embargo, estudios hidrogeológicos (GIAI, 2005) muestran la existencia de acuíferos en el área de estudio y cercanos a ella (Figura 2) ${ }^{12}$. La Formación Santa Isabel es la más importante, pero debido a la presencia de "sales evaporíticas en cantidades importantes" (GIAI, $2005,27)$ sus aguas son salobres. El otro acuífero cercano es El Meauco localizado en el centrooeste de la provincia con presencia de agua dulce. La recarga del acuífero Santa Isabel está sujeta a las escasas lluvias, la presencia de torrentes secos (activos en épocas de precipitaciones) localizados en el extremo oeste y se supone que las infiltraciones del Atuel también lo alimentarían.

El consumo de agua en el sur mendocino sobre el límite con La Pampa, está orientado a satisfacer la producción frutivinícola. Por otro lado en el tramo medio del río Atuel dentro de la provincia cuyana, se suma otro uso ligado al turismo. La zona del cañadón del Atuel es uno de los lugares donde se practican deportes y actividades recreativas acuáticas como rafting y canotaje. Las poblaciones más importantes aquí son San Rafael, General Alvear y Malargue. La población total es de 260.678 habitantes ${ }^{13}$.

A los actores demandantes de agua pampeanos, ligados a la producción de subsistencia, debemos sumar los actores de Mendoza. La disparidad en términos económicos y poblacionales es evidente. El sudeste mendocino tiene una población mayor y con niveles económicos altos. Por lo que, las demandas y reclamos que estos también realizan, se efectúan desde una posición de poder y, por consiguiente, de presión mayor a la de los pampeanos. La demanda del sudeste mendocino se genera ante la necesidad de mantener y aumentar la producción vinícola orientada a la exportación, principalmente, con más riego. No obstante, en las épocas de sequías en que no

\footnotetext{
9 Para mayor información se puede ingresar al trabajo disponible online en: http://www.lapampa.gov.ar/images/stories/Archivos/RecursosHidricos/CuencaDesaguaderoSalado/Evolu cion_de_la_Poblacion.pdf

${ }^{10}$ Capa de agua subterránea.

${ }^{11}$ Para ampliar información estadística se sugiere visitar el sitio de la Secretaría de Recursos Hídricos de la Provincia de La Pampa: http://www.bdh.lapampa.gov.ar/aguasubterranea_listado.php?xgap historial=reset.

12 Este esquema fue extraído del trabajo "Estudio de las aguas subterráneas en el oeste de la provincia de La Pampa" impulsado por el Consejo Federal de Inversiones con el objetivo de conocer las disponibilidades de agua.

13 Puede obtenerse más información en la página de INTA (Instituto Nacional de Tecnología Agropecuaria) en relación al Proyecto Regional con Enfoque Territorial "Desarrollo del oasis sur de la provincia de Mendoza" (2013-2019), disponible en http://inta.gob.ar/proyectos/MZASJ-1251306 . En esta página se puede acceder a la información de los distintos talleres y seminarios realizados en el marco de dicho proyecto.
} 
se han registrado suficientes nevadas, los deshielos son escasos. Ante el reclamo pampeano se argumenta que primero deben regar sus cultivos para luego dejar el agua correr. Su fundamento se asienta en el fallo de la Suprema Corte de Justicia de 1987. Si bien la corte dictaminó la interprovincialidad del río Atuel, Mendoza tiene el derecho de regar unas 75.600 ha. y sus excedentes son los que se liberan agua abajo del río.

FIGURA 2

Perfil con las formaciones geológicas y existencias de aguas subterráneas, paralelo $36^{\circ} 05$

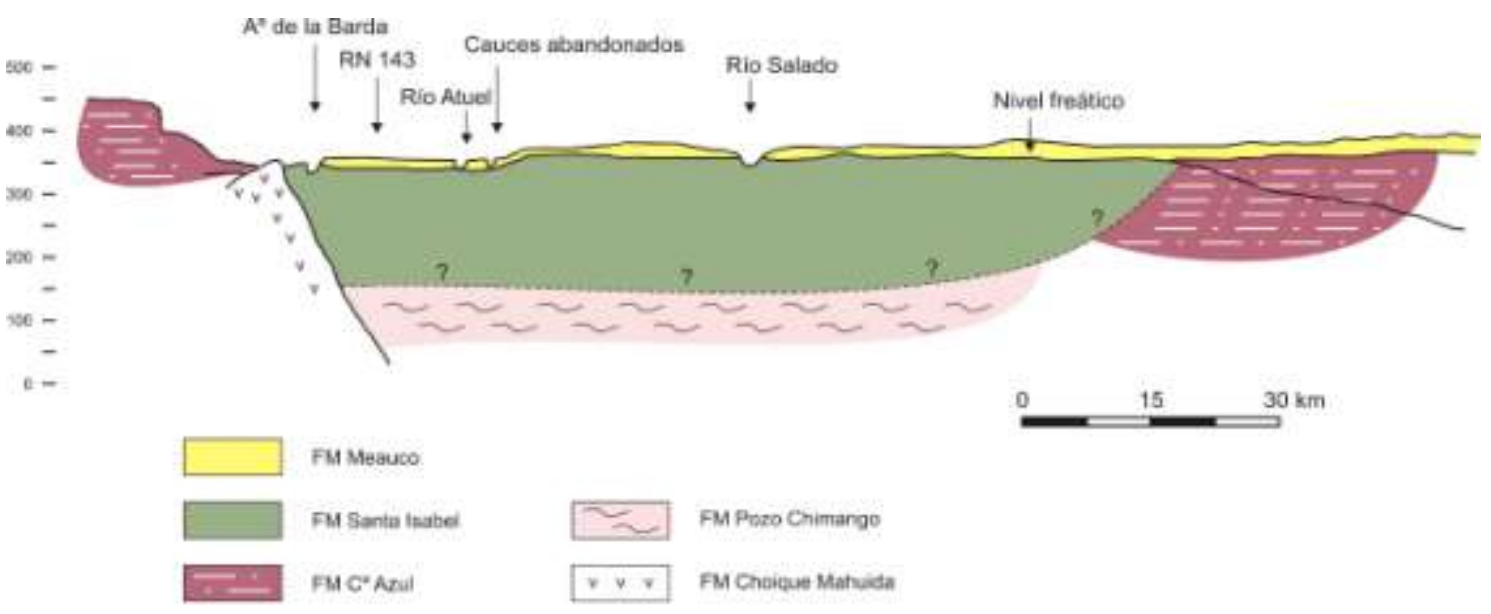

Fuente: Estudio de las Aguas Subterráneas en la Región Oeste de la Provincia de La Pampa (Lic. GIAI, CFI).

\subsection{EL CASO DEL RÍO ATUEL COMO EJEMPLO DE CONFLICTO POR EL ACCESO AL AGUA}

Las características presentadas hasta aquí definen un espacio que ante una intervención antrópica desaprensiva se desestabiliza y, en el caso del acceso al agua, se generan conflictos. En el oeste pampeano ello se produjo ante la intervención del río Atuel en la provincia de Mendoza por diversos usos y su caudal comenzó a disminuir paulatinamente. Este conflicto es de larga data dado que hay antecedentes de las mermas en el caudal fluvial en las primeras décadas del siglo XX (DIFRIERI, 1980).

Por otra parte, las indefiniciones en el marco jurídico territorial hasta 1951, año en que La Pampa se transformó en provincia, favorecieron las intervenciones inconsultas del agua tanto por parte de Mendoza como del Estado nacional. En el caso de Mendoza fue una de las primeras catorce provincias del país, razón por la cual ya contaba con una estructura gubernamental y con población permanente que realizaba diversas actividades productivas.

A fines del siglo XIX en Mendoza se comenzó a impulsar la actividad vitivinícola lo que transformó paulatinamente la economía de la provincia (JORBA, 2000) y contribuyó a la consolidación de los oasis de riego ${ }^{14}$. Las parcelas se delimitaron a partir de los primeros desvíos de brazos del río, en algunos casos en forma clandestina especialmente en las poblaciones sureñas cercanas al límite con el nuevo territorio.

Paralelamente, desde el gobierno nacional y ante demandas de los sucesivos gobernadores territoriales, se buscó fomentar la ocupación espacial del oeste pampeano semiárido. En tal

${ }^{14}$ DIFRIERI en su libro "Historia del Atuel” afirma que " Hacia 1885, existían en Mendoza, en el entonces departamento de Malargue 100 hectáreas cultivadas con agua de riego extraída del río Atuel (...) en 1890, se hallan mensuradas parcelas bajo riego con una superficie total de casi 1200 kilómetros cuadrados o sea 120.000 ha. de riego posible, con canales derivados exclusivamente del río Atuel" (DIFRIERI, 1980, 20). 
sentido, se mensuraron los primeros terrenos para la creación de colonias pastoriles. Surgen así, poblaciones como Colonia Butalo entre los años 1905 y 1910, localizada en las cercanías del brazo del Atuel llamado Butalo, con una superficie de 10.000 ha subdividida en lotes de 100 ha., junto a la colonia Emilio Mitre situada al este de la localidad de Santa Isabel y del área de los ríos, donde se establecieron las poblaciones remanentes del pueblo Ranquel. Sin embargo, tanto por la falta de construcción de infraestructura como por la disminución de los caudales del río, la Colonia Butalo se despobló en la primera mitad del siglo. Su población emigró ${ }^{15}$ a otras localidades dentro de la provincia o al sur de Mendoza. En estos años, los cortes de los brazos del río se producían tanto por desvíos clandestinos como por taponamientos, como en el caso del "tapón de Ugalde" en la zona de Loncovaca, originando reclamos por parte de los pobladores y el gobernador pampeano.

En 1947 bajo el gobierno de Juan Domingo Perón se construyó la represa El Nihuil sobre el curso superior del río, ello significó el corte total del mismo. La pérdida del agua en el oeste pampeano significó la expulsión de población de los pequeños poblados y la destrucción de una extensa zona de humedales de los "Bañados del Atuel". Bajo el mismo gobierno el territorio pasó a ser provincia, con este nuevo estatus se iniciaron paulatinamente las demandas contra Mendoza, básicamente y también contra el Estado nacional.

En este proceso de lucha por el agua se puede establecer como un hito el año 1987 cuando la Corte Suprema de Justicia dictaminó que el Atuel es un río interprovincial, por lo tanto la provincia de Mendoza debía liberar una determinada cantidad de agua para que ingrese en el oeste pampeano, luego de regar 75.600 ha., además de mejorar la eficiencia del riego por medio de obras. Desde entonces los conflictos y enfrentamientos se han hecho más agudos entre ambas provincias, dado que Mendoza desconoce al Atuel como un río interprovincial argumentando que el río muere antes de ingresar en La Pampa y que la provincia sureña no tiene necesidades de uso del agua en el oeste debido a la escasa población y uso del suelo agrícola.

En las últimas dos décadas, la lucha por el agua se ha transformado en una demanda popular (ROJAS y WAGNER, 2016), sobre todo por los pobladores del oeste, donde se incluyen tanto a los habitantes de las localidades y parajes como a los puesteros, quienes han crecido, en muchos casos, en un ambiente totalmente árido, lo que les ha permitido desarrollar una conciencia del agua a partir de su escasez ${ }^{16}$. Los distintos gobiernos provinciales de La Pampa han acompañado las demandas con diversos éxitos, incorporando la cuestión del acceso al agua en una política del Estado provincial.

\subsection{LOS NUEVOS APORTES EN TORNO A LOS ASPECTOS DEL CICLO HIDRO- SOCIAL PAMPEANO}

Hasta aquí se detectaron las características del conflicto como así también, la caracterización del territorio hidro-social del oeste pampeano. No obstante, como se planteó en la introducción al trabajo, para comprender como ha sido el proceso del conflicto, es necesario analizar los aspectos que, entendemos, componen el ciclo hidro-social en la zona de estudio.

Las fuentes trabajadas hasta el momento permiten identificar diversos aspectos que interactúan y se complejizan a través del tiempo. La circulación del agua en el oeste pampeano en tiempos prehistóricos es comprobable, tanto por las marcas en el relieve (cauces y área de humedales marcada en la zona de ingreso a La Pampa), como en la existencia de asentamientos humanos cerca de las riberas y en los testimonios de la época colonial que han quedado registrados. El ciclo hidro-social comenzó a modificarse cuando surgieron nuevos intereses

\footnotetext{
${ }^{15}$ La emigración que se produjo en el oeste pampeano luego del corte del río, fue rural y se dirigió hacia los centros poblados del sur mendocino o del este pampeano. El nombre que se le dio fue el de "diáspora saladina", estos pobladores perdieron todos sus animales y debieron abandonar sus tierras. Acabaron instalándose en las ciudades como Victorica, General Acha, Santa Rosa convirtiéndose en mano de obra no calificada.

${ }^{16}$ Esta conciencia de la ausencia del agua ha contribuido a crear un imaginario del agua a nivel provincial, así como bandera de lucha se esgrime el eslogan "río robado" y desde el gobierno de la provincia en el año 2001 se elaboró junto a músicos y poetas provinciales, el "Cancionero de los ríos".
} 
económicos que supusieron nuevos usos. En este caso Mendoza con el desarrollo de la producción frutivinícola y la gestión del agua para crear áreas de oasis en una zona semidesértica. Esta lógica económica y de producción se dio en un contexto de crecimiento poblacional y ocupación de nuevos territorios por el Estado nacional que estaba consolidándose, generó, de este modo, desequilibrios en el acceso al agua en el caso de esta zona. La Pampa paso a ser uno de los nuevos territorios nacionales al que no se le otorgó impulso desde el Estado y su problema hídrico se volvió recurrente.

En este contexto es que se agudizó la problemática en torno al acceso al agua en un espacio semiárido como el oeste pampeano, la cual fue profundizándose a lo largo del siglo XX y continúa sin resolución en la actualidad. Los aspectos que se decantan de este proceso son: los actores, las representaciones en torno al agua, las tensiones sociales, los conflictos y las características del territorio hidro-social (Figura 3).

FIGURA 3

Representación del ciclo hidro-social en el oeste pampeano

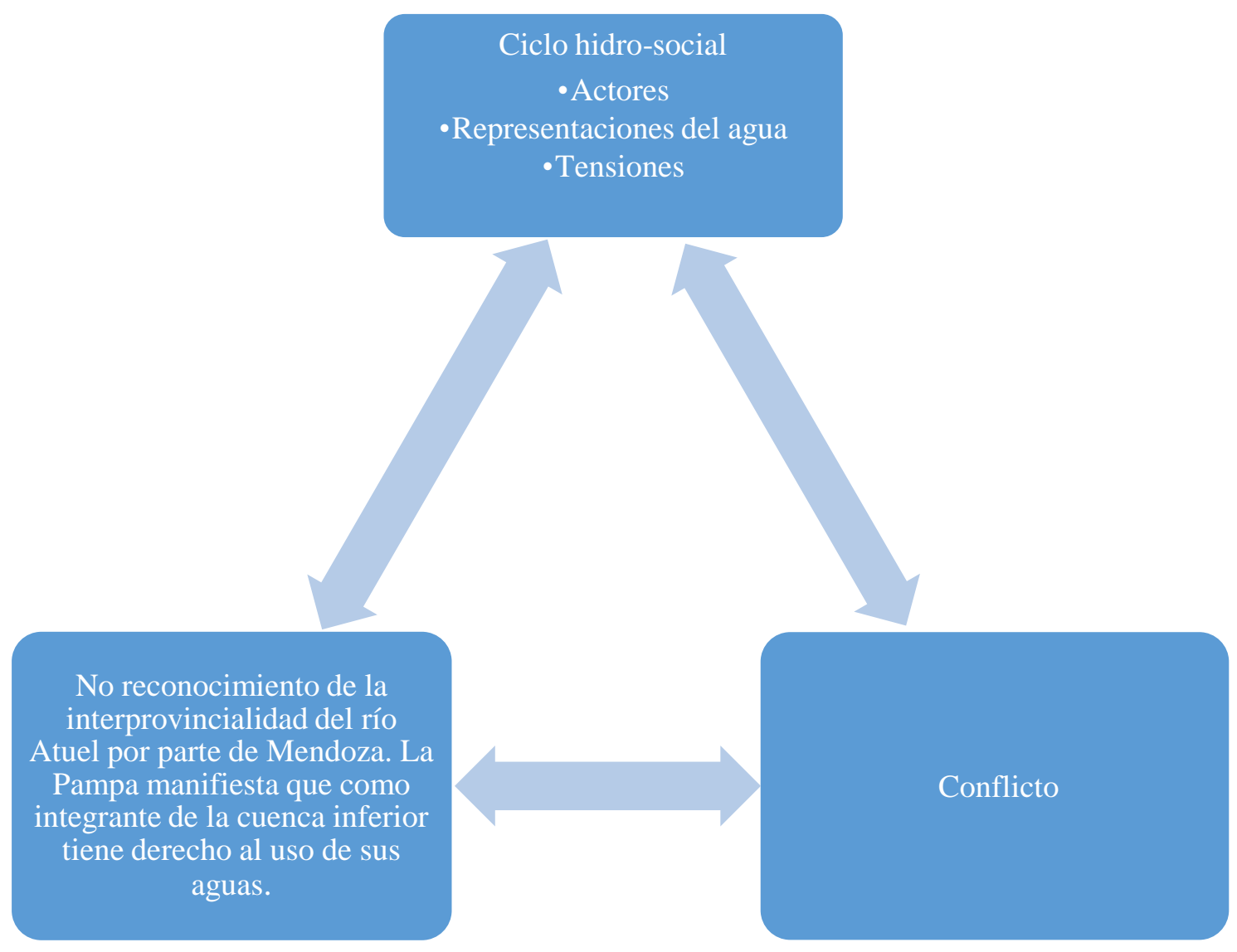

Fuente: Elaboración propia.

Los actores dentro de este ciclo tienen diferentes dimensiones y grado de intervención en el conflicto. Son el sujeto colectivo con identidad propia que, a partir de la tenencia de ciertos recursos, actúa dentro de una sociedad en defensa de los intereses de los miembros que la componen para dar respuestas a necesidades concretas. Por un lado, están los que se ven afectados inmediatamente ante la falta de circulación del río, como en el caso de los puesteros, los productores (pequeños y medianos) y los habitantes de las localidades de Algarrobo del Águila y Santa Isabel. Ellos son los que manifiestan sus primeros reclamos que hacen llegar a las autoridades nacionales por medio del gobernador del territorio. En tanto por otro lado, se encuentran las organizaciones intermedias (asambleas, técnicos, profesionales) y el Estado provincial que participan por medio de sus conocimientos, la mediación y reclamo directo a los 
gobiernos provinciales como nacional para lograr una solución. En el caso del Estado especialmente, éste puede actuar como mediador a través de legislación, control o gestión del agua.

Otro de los componentes del ciclo hidro-social son los imaginarios en torno al agua. Los imaginarios o representaciones creados en relación al agua son subjetivos de acuerdo a cada actor social. Varían desde la sacralización, identificación con los territorios hídricos, la interrelación agua-lugar hasta valorizar al agua como una mercancía y un recurso manipulable por medio de distintas estrategias

FIGURA 4

Cronología del desarrollo de las tensiones

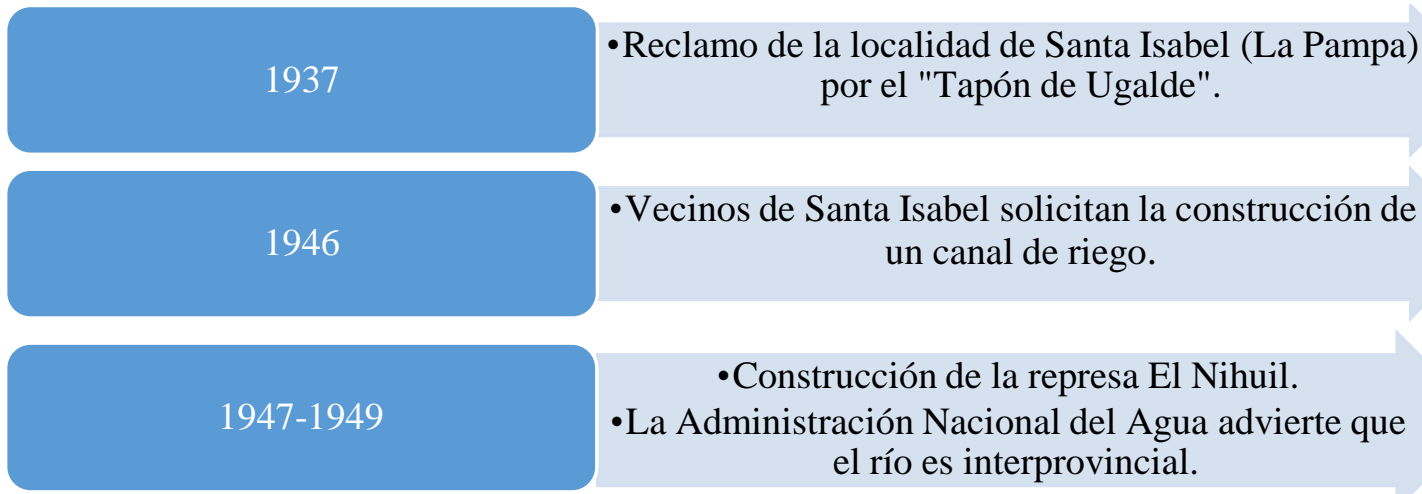

-Mendoza rechaza la interprovincialidad del Atuel.

1979

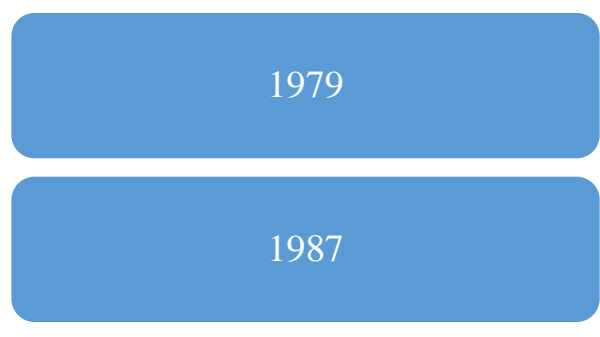

2008-2016
- Fallo de la Suprema Corte de Justicia declara la interprovincialidad del río Atuel.

-Aumento de las demandas y reclamos por el río Atuel de parte de La Pampa.

Fuente: Elaboración propia

En cuanto a las tensiones (Figura 4), son aquellas situaciones emergentes previas a un conflicto o indican que este se encuentra latente. Dichas situaciones denotan fricciones y luchas entre las relaciones de poder de los distintos actores involucrados. En este caso se puede establecer una cronología donde los puntos de mayor tensión están representados por los reclamos de los pobladores y del gobierno pampeano. En el año 1921 se comenzó a desarrollar la colonia de Carmensa en Mendoza hecho que produjo el primer desvió de las aguas del Atuel. En 1937 se obstaculizó la circulación de agua nuevamente, ello produjo reclamo de los vecinos de Santa Isabel por el "Tapón de Ugalde" en el paraje Loncovaca ya sobre el límite interjurisdiccional. La proyección y posterior construcción de la represa del Nihuil en la década del cuarenta creo nuevas fricciones. En 1942 los habitantes de Santa Isabel piden la construcción de un canal de riego necesario para el lugar, pero no es factible por hallarse en curso el proyecto de la represa del Nihuil. En tanto entre 1948-49, comienza a advertirse desde la Administración Nacional del Agua de que las aguas son interprovinciales, para ese momento ya se hallaba en funcionamiento la represa El Nihuil. Luego de la provincialización de La Pampa (año 1951) los reclamos por el río se volvieron parte de la agenda política. En el año 1979 Mendoza rechazó que el Atuel sea interprovincial. Como consecuencia se produjo la judicialización del conflicto. En 1987, la Suprema Corte de Justicia establece que el río es interprovincial y que Mendoza debe realizar una suelta anual de las aguas, ello no se cumple. 
Paralelamente se fue fortaleciendo una conciencia ambiental que cimentó la organización de asambleas y respaldó la demanda que se hizo más intensa por gran parte de la población pampeana. En este marco entre los años 2008 y 2016 aumentaron los reclamos pampeanos en el contexto de la lucha por los bienes comunes, en este caso el agua.

El no reconocimiento de la interprovincialidad del río y su consecuente uso de forma exclusiva por Mendoza, conduce al conflicto. En tal sentido tomando a ROBBINS (1994) como referente, el conflicto se produce cuando una de las partes involucradas percibe que otra la afectó o puede afectarla de forma negativa. Los conflictos se inician tanto por factores externos como internos que actúan como disruptores. En esta situación se puede generar una reacción de cambio o reforma del sistema imperante hasta ese momento.

Por último, el territorio hidro-social es el aspecto que otorga el contexto donde se desarrolla el ciclo hidro-social. Es el resultado de una configuración espacial de personas, instituciones, flujos de agua, tecnología hidráulica y el ambiente bio-físico, los cuales giran en torno al control del agua (BOELENS et al., 2016) y se han ido configurando a través del tiempo.

\section{CONSIDERACIONES FINALES}

A nivel mundial, el acceso al agua potable, es uno de los problemas que mayor demanda atención, estudios y acción por parte de la población y gobiernos. La intensidad de las actividades extractivas y sus consecuencias (contaminación y derroche) bajo el modelo capitalista son una de las mayores amenazas para lograr una distribución equitativa de este recurso.

La necesidad de comprender cómo fluye el agua en medios urbanos y rurales y bajo qué redes de poder, hace necesario que se considere a la circulación del agua en la naturaleza ya no mediada sólo por cuestiones físicas (clima, relieve, etc.) sino por elementos sociales que dirimen las relaciones de poder en torno al agua apelando a diversas estrategias que abarcan desde lo legal a lo cultural, configurando un ciclo hidro-social. Aquí también se pueden diferenciar y analizar esos elementos constitutivos: actores, tensiones, conflictos, representaciones y territorios hidro-sociales.

El caso del río Atuel en La Pampa es un exponente de esta problemática y de la posibilidad de comenzar a comprender los flujos de agua como flujos de poder, no sólo como un elemento más de la naturaleza ajeno a la intervención humana. La comprensión y utilización del concepto de ciclo hidro-social puede ayudar a entender los conflictos en torno al agua, como así también a considerar los territorios desde una perspectiva más amplia que excede a los límites jurisdiccionales y escucha e integra las demandas de los habitantes del lugar. Una alternativa constructiva es este aspecto, sería que las asambleas pampeanas y del sur mendocino dialogaran en calidad de habitantes y pobladores de un espacio semiárido donde, si bien para los pampeanos el acceso al caudal del Atuel está negado, los pobladores mendocinos tampoco lo tienen asegurado, sobre todo en este contexto de cambio climático y recrudecimiento de actividades extractivas que involucran grandes cantidades de agua dulce.

En estas geometrías de poder los actores participantes deben aprehender el territorio hidrosocial desde una óptica que tienda a la distensión y el diálogo para distribuir el agua equitativamente entre dos provincias. De este modo tratar de restituir al río Atuel su antigua dinámica de circulación.

En este caso donde el territorio hidro-social excede los límites provinciales, debería pensarse en cómo articular las demandas de agua tanto para Mendoza como La Pampa. En tal caso sería necesario que los actores afectados directamente como pequeños y medianos productores mendocinos, puesteros y pobladores de las localidades oesteñas, constituyan un ámbito de diálogo para movilizarse y direccionar sus reclamos. La continuidad del conflicto lleva en múltiples ocasiones, al enfrentamiento entre ellos.

En relación con lo expuesto, es necesario, que en la búsqueda de una solución al conflicto hídrico, se comience a pensar y actuar ya no desde un posicionamiento netamente provincial, sino hidro-territorial. Los ríos generalmente, y en este caso en particular, atraviesan o son compartidos por varias provincias realizándose diversos usos. En tal sentido debe gestionarse el manejo de las cuencas desde una perspectiva eco política. Es decir, propiciar el diálogo y 
encuentro entre las comunidades afectadas directamente que interaccionan en la parte final de la cuenca del Atuel. Además de considerar las dinámicas naturales de aumento de caudales, desbordes, períodos secos, junto a los espacios ecológicos que nutren.

\section{AGRADECIMIENTOS}

Este trabajo forma parte del proyecto "Aplicación de Tecnologías de la Información Geográfica al Estudio Integral y Comparativo de Problemáticas Ambientales” (2016-2018), financiado por la Secretaría General de Ciencia y Técnica (SGCyT) de la Universidad Nacional del Sur.

\section{BIBLIOGRAFÍA}

AUSTRAL, A. (1971): "El yacimiento arqueológico de Vallejo, NO de la provincia de La Pampa. Contribución a la sistematización de la prehistoria y la arqueología de la región pampeana". Relaciones. Vol N5, p.49-70.

BERÓN, M. (2008): "Puelches: una historia que fluye junto al Salado. Arqueología de los departamentos Curaco y Lihue Calel. Una historia de cazadores". En: Salomón Tarquini, Laguarda y Kuz (Ed.), Santa Rosa, EdUNLPam, p.16-34.

BOELENS, R.; HOOGESTEGER, J.; SWYNGEDOUW, E.; VOS, J. y WESTER, P., (2016): "Hydro-social territories: a political ecology perspective" en: Water International. Disponible en: http://dx.doi.org/10.1080/02508060.2016.1134898 [Consulta: 01 de agosto de 2016]

BOELENS, R. (2015): Water justice in Latin America. CEDLA, Amsterdam, 46 pp.

BRUNIARD, E., (1982): "La diagonal árida argentina: un cambio climático real". Revista Geográfica. Vol. N ${ }^{\circ}$ 95, p.1-20.

CAZENAVE, W., (1997): "La desaparición de los Bañados del río Atuel: un caso de acción antrópica negativa". Huellas. Vol. $\mathrm{N}^{\circ} 2$, p. 28-37.

DIFRIERI, H., (1980): Historia del Río Atuel. UBA, Buenos Aires, 45 p.

DIFIRIERI, H., (1981): Evolución del poblamiento del área de influencia del Atuel en territorio de La Pampa. Extra Producciones, Santa Rosa, 25 p.

ESCOBAR, A., (2010): Territorios de diferencia: lugar, movimiento, vida, redes. E. Editores, Popayan, $222 \mathrm{p}$.

GIAI, S., (2005): Estudio de aguas subterráneas en la Región Oeste de la provincia de La Pampa. Informe final. Tomo I. Consejo Federal de Inversiones, Santa Rosa.

GOBIERNO DE LA PAMPA, (s/f): "Evolución de la población en el área de la cuenca del río Salado-Chadileuvú-Curacó (Provincia de La Pampa). Análisis expeditivo del período 19202007".

Disponible

en: http://www.lapampa.gov.ar/images/stories/Archivos/RecursosHidricos/CuencaDesaguaderoS alado/Evolucion de la Poblacion.pdf. [Consulta: 16 de julio de 2017].

JORBA, R., (2000): "Cambios tecnológicos y transformaciones económico-espaciales en la vitivinicultura de la provincia de Mendoza (Argentina), 1870-2000". Scripta Nova. Revista Electrónica de Geografía y Ciencias Sociales. Vol. 83, Nº 69.

ILLICH, I. (1974): Energy and equity. Harper \& Row, Nueva York, 84 p.

IVARS, J.D., (2015): "Mercados mundiales y racionalización del uso del agua en Mendoza, (Argentina)". Ánfora. Vol. 22, pp. 71-97.

LARSIMONT, R., (2014): "Ecología política del agua: reflexiones teórico-metodológicas para el estudio del regadío en la provincia de Mendoza". Disponible en: http://www.ina.gob.ar/ifrh-2014/Eje1/1.03.pdf . [Consulta: 23 de junio de 2016].

LINTON, J., (2010): What is Water? The History of a Modern Abstraction. UBC Press, Vancouver, $333 \mathrm{p}$.

LUO, .T. YOUNG, R.; REIG, P. (2015): “Aqueduct projected water stress country rankings". Disponible en: http://www.wri.org/publication/aqueduct-projected-waterstresscountryrankings?utm campaign=WRIAqueduct\&utm source=blogpostgraphic\&utm medium=image . [Consulta: 14 de marzo de 2016]. 
MEDUS, N.; PODUJE, M.I. (1997): Artesanos tradicionales de La Pampa. Gobierno de La Pampa, Santa Rosa.

MERLINSKY, G., (2013): Cartografías del conflicto ambiental en Argentina. Ed. Ciccus, Buenos Aires, $320 \mathrm{p}$.

MOROTE, A., SAURÍ, D. y HERNANDEZ, M., (2016): "Residential tourism, swimming pools and wáter demand in the Western Mediterranean". The Professional Geographer. Vol. 69, pp. 1-11.

MOROTE SEGUIDO, A., (2016): "La disminución del consumo de agua urbano-turística en la costa de Alicante (España): una amalgama de causas múltiples e interrelacionadas". Revista de Estudios Regionales. N¹06, pp. 133-164.

OLCINA CANTOS, J. y VERA-REBOLLO, J.F. (2016a): “Adaptación del sector turístico al cambio climático en España. La importancia de las acciones a escala local y en empresas turísticas". Anales de Geografía de la Universidad Complutense. $N^{\circ} 36$ (2), pp. 321-352.

OLCINA CANTOS, J. y VERA-REBOLLO, J.F. (2016b): "Cambio Climático y política turística en España: Diagnóstico del litoral mediterráneo español”. Cuadernos de Turismo. No38, pp. 323-359. Disponible en: http://www.redalyc.org/pdf/398/39848506014.pdf [Consulta: 27 de junio de 2017]

ORGANIZACIÓN DE NACIONES UNIDAS, (2007): "Cambio climático y diversidad biológica", Disponible en: https://www.cbd.int/doc/bioday/2007/ibd-2007-booklet-01-es.pdf [Consulta: 23 de junio de 2016].

PERALES MIRANDA, V. H. (2010): "Conflictos geopolíticos por el agua en las cuencas mineras del Departamento de Oruro, Bolivia”. Disponible en: https://vertigo.revues.org/9769 [Consulta: 16 de marzo de 2016].

PODUJE, M. I. (2004): "Las investigaciones arqueológicas en la provincia de La Pampa". Quinto Sol. Vol. N ${ }^{\circ}$, pp. 135-145.

ROBBINS, S. y Judge, T., (1994) Comportamiento organizacional, conceptos, controversias y aplicaciones". Prentice Hall Hispanoamericana, México, 780 p.

ROJAS, F.; WAGNER, L., (2016): "Conflicto por la apropiación del río Atuel entre Mendoza y La Pampa (Argentina)”. Halac. Vol IV, N², pp. 278-297.

SWYNGEDOUW, E., (2009): "The political economy and political ecology of the hydro-social cycle". Journal of Contemporary Water Research \& Education. Vol. $\mathrm{N}^{\circ} 142$, 2009, pp. 5660.

SWYNGEDOUW, E. (2011): “iLa naturaleza no existe! La sostenibilidad como síntoma de una planificación despolitizada". Urban. $\mathrm{N}^{\circ} 1$, pp. 41-66.

SWYNGEDOUW, E. (2013): "Into the sea: desalination as hydro-social fix in Spain". Annals of the Associations of American Geographers, Vol. 103, pp. 261-270.

SWYNGEDOUW, E. (2015): Liquid power. Contested hidro-modernities in Twentieth Century in Spain, 1898-2010. MIT Press, Londres, 301 p.

YACOUB, C.; DUARTE, V.; BOELENS, R., (2015): Agua y ecología política. El extractivismo en la agro exportación, la minería y las hidroeléctricas en América Latina. Abya-Yala, Quito, 308 p. 